\title{
One-step twin-screw extrusion process of cellulose fibers and hydroxyethyl cellulose to produce fibrillated cellulose biocomposite
}

\author{
Hesam Taheri $\cdot$ Maiju Hietala $\cdot$ Kristiina Oksman $\mathbb{D}$
}

Received: 15 October 2019/Accepted: 12 June 2020/Published online: 28 July 2020

(C) The Author(s) 2020

\begin{abstract}
In this work, the defibrillation of cellulose fibers (CF) in the presence of hydroxyethyl cellulose (HEC) within the one-step twin-screw extrusion (TSE) process was examined. The effect of the TSE on cellulose fiber size reduction as well as CF-HEC biocomposites properties were investigated. The results showed that the TSE of cellulose fiberhydroxyethyl cellulose (CF-HEC) with different cellulose fiber contents $(50,65$, and $80 \mathrm{wt} \%)$ resulted in partial defibrillation of the cellulose fibers. The fractionation test of the cellulose fibers confirmed that
\end{abstract}

Electronic supplementary material The online version of this article (https://doi.org/10.1007/s10570-020-03287-3) contains supplementary material, which is available to authorized users.

H. Taheri

Fiber and Particle Engineering Research Unit, Faculty of Technology, University of Oulu, P.O. Box 4300,

90014 Oulu, Finland

e-mail: hesam.taheri@oulu.fi

\section{Hietala}

Division of Materials Science, Department of Engineering Sciences and Mathematics, Luleå University of

Technology, 97187 Luleå, Sweden

e-mail: maiju.hietala@oulu.fi

K. Oksman $(\bowtie)$

Mechanical and Industrial Engineering (MIE), University

of Toronto, 5 King's College Road, Toronto,

ON M5S 3G8, Canada

e-mail: kristiina.oksman@Itu.se their size was reduced and some fibrillation was observed in microscopy studies. The maximum width reduction of $46 \%$ occurred with 80 wt $\%$ cellulose content. However, the partial width reduction was also observed with $50 \%$ and $65 \mathrm{wt} \%$ of cellulose contents. Based on rheological measurements, the shear-viscosity trend of CF-HEC dispersion abruptly dropped when higher fiber content ( $80 \mathrm{wt} \%)$ was extruded, which was related to the fibrillation of the cellulose fibers as well as the reduction of the length. The extruded CF-HEC materials (powder form) were compression molded to prepare the biocomposites with different cellulose fiber contents $(50,65$, and 80 wt $\%)$. The extruded CF-HEC powders were diluted with addition extra HEC to make biocomposites with lower fiber content $(20 \%, 30 \%$, and $40 \mathrm{wt} \%)$ and compression molded to study how the size reduction of the cellulose fibers affected the mechanical properties of biocomposites. The results showed that the E-modulus improved from $0.4 \mathrm{GPa}$ of the neat HEC to $1.6 \mathrm{GPa}$ for the composite with $40 \mathrm{wt} \% \mathrm{CF}$. Interestingly, the tensile strength of CF-HEC biocomposite with $40 \mathrm{wt} \%$ confirmed a clear improvement from 9.8 to $26.6 \mathrm{MPa}$, confirming good interaction between $\mathrm{HEC}$ and $\mathrm{CF}$. 
Graphic abstract Preparation (mixing, TSE, and hot-pressing) and characterization (FE-SEM, rheometry, and tensile test) of CF-HEC biocomposite

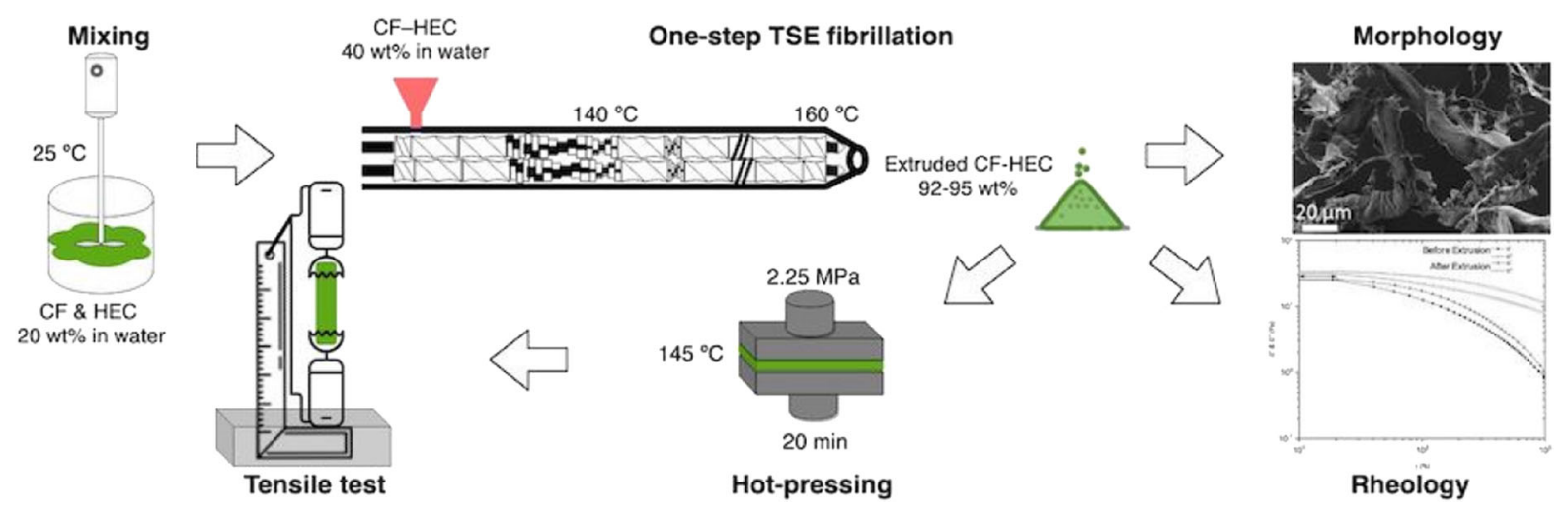

Keywords Twin-screw extrusion (TSE) . Defibrillation · Cellulose fiber (CF) - Hydroxyethyl cellulose (HEC) · Biocomposite

\section{Introduction}

The annual biomass production of cellulosic materials is more than 1 trillion tons (Siró and Plackett 2010), with an unexhausted source that can be replaced by photosynthesis without affecting feedstock. The wide variety of bio-based materials from different resources and their availability in many forms provide a broad element that can be used for different applications. Nowadays, fibrillated cellulose biocomposites are in the spotlight due to their extraordinary features, such as physical, chemical (Foster et al. 2018), mechanical (Hietala et al. 2011a), and optical properties (Simão et al. 2015) and also due to the availability of raw sources, such as wood, wood pulps and rejected or side-products of industry (Wu et al. 2018). In this regard, there is a high propensity to increase the proportion of renewable resources in fiber-polymer matrix biocomposites. However, poorly distributed micro- and nanoscale fiber reinforcements and the weak fiber-polymer interaction result in the decrement of the biocomposite's mechanical property (Berglund and Peijs 2010). In cellulose fiber (CF), the cellulose microfibrils are the reinforcement elements of the plant, which are embedded in a complex matrix like lignin-hemicellulose (Toba et al. 2012). Likewise, the cellulose fibers (CF) (e.g., micro- and nanofibrillated fibers) can be used as reinforcing elements in biocomposites after the extraction of lignin and hemicelluloses. Therefore, the overall scenario of cellulose-based biocomposites can be focused on the defibrillation of cellulose fibers and their suitable dispersion or distribution in a new (bio)polymer matrix (Osong et al. 2016).

Various processing methods have been used to extract cellulosic materials into different micro- and nanoscale morphologies. The processing of microand nanoscale cellulosic materials, such as CNF, can be done mostly with grinding (Iwamoto et al. 2007; Lahtinen et al. 2014; Nechyporchuk et al. 2015), homogenization/microfluidization (Ho et al. 2011; Taheri and Samyn 2016). The twin-screw extrusion (TSE) method has also been used (Hietala et al. 2014). As the energy consumption of grinding and microfluidization methods is very high (Spence et al. 2011; Josset et al. 2014), few scientific works have been focused on TSE to defibrillate CFs (Hietala et al. 2011b; Ho et al. 2015; Rol et al. 2017). However, none of these works have yet been successful, and in some cases the defibrillation of fibers can occur with a high number of passes within the extruder. Defibrillation of CFs by TSE is an effective method to disintegrate the CFs. However, a higher number of passes within the extrusion can result in fiber degradation/breakage.

Due to the poor thermoformability of CFs and strong hydrogen bonds, the CFs alone cannot be used for continuous melt processing. Moreover, the hydrophilic behavior of CFs causes some dispersive or 
distributive issues when they are processed with a hydrophobic polymer or biopolymer matrix (Hietala et al. 2014). However, the non-soluble CFs that are derivatized by several methods, such as esterification (Fox et al. 2011) and carbamation (Sirviö and Heiskanen 2017), turn into a water-soluble semisynthetic polymer. Therefore, cellulose derivatives can show better formability and compatibility with $\mathrm{CFs}$ and can be a suitable matrix for $\mathrm{CFs}$ in continuous biocomposite processing, such as melt extrusion. In this regard, CFs can act as reinforcements in the watersoluble semi-synthetic matrix when the cellulose derivative matrix does not have desirable mechanical properties for the material applications.

Hydroxyethyl cellulose (HEC) is one of the nonionic cellulose derivatives, which can be used in different applications, such as textiles, paperboard, paints, adhesive, and the food industry, (Loner and $\mathrm{Oh}$ 2001; Rahmat et al. 2011) and also in pharmaceuticals as binders, stabilizers, thickeners, and film formers (Kamel et al. 2008). However, it is the least studied cellulose derivative compared to hydroxypropyl cellulose and hydroxypropylmethyl cellulose (HPMC), and most of the published works are related to the mixture of HEC and other polymers (Morales and McConville 2011). There are some works about CFs and HEC (Sehaqui et al. 2011; Hooshmand et al. 2017); however, the core of these works is related to the mixture of cellulose nanofibers and HEC as a nanocomposite.

Other materials with higher compatibility with CFs have also been used to improve the amount of fibrillation within the TSE process. Starch was used as one of these materials due to the water-soluble behavior and compatibility with cellulose within the TSE process (Hietala et al. 2013; Cobut et al. 2014). However, the higher fiber contents led to some aggregations of CF within the TSE process, and the strength was less improved due to the lubricating effect of starch (Hietala et al. 2013). In this regard, HEC, due to its compatibility with cellulose fibers, can also be a proper matrix for TSE processed with CF to elucidate the level of defibrillation and interaction of $\mathrm{CF}$ with the HEC matrix within the one-step twinscrew extrusion process.

Because the higher number of passes within extrusion results in the degradation of CFs (Ho et al. 2015), the one-step twin-screw extrusion method might decrease the fiber degradation or the decrement of crystallinity. Moreover, a higher number of passes within extrusion might not be interesting for an industrial application where continuous processing is performed. However, the CF-HEC biocomposite obtained by simultaneous extrusion of CF and HEC (one-step TSE) does not need to be redispersible to be used in continuous industrial applications. On the one hand, the twin-screw extrusion (TSE) method can be an alternative method for pelletizing fibrillated CFs and different polymer matrices (Suzuki et al. 2013) or biopolymeric materials (Hietala et al. 2011b; Hietala and Oksman 2018; Haque et al. 2018). On the other hand, the fibrillation of the CFs and the mixing of the fibers and biopolymer matrix can be improved at the same time with the use of TSE.

In this study, the defibrillation of CF in presence of HEC matrix was examined by using different $\mathrm{CF}$ contents, and the CF-HEC biocomposites were produced by the one-step TSE method. To take the positive credit from TSE with the minimum fiber degradation/breakage and without repeating the extrusion steps, the one-step TSE processing of the fiber/matrix was investigated due to fiber defibrillation and mixing the fibers with the matrix at the same time. The one-step extrusion method resulted in the partial fibrillation and size reduction of cellulose fibers, and at the same time leading to the CF-HEC biocomposite with high cellulose content. The extruded CF-HEC and the diluted extruded CF-HEC were hot-pressed to observe how the extrusion process can be affected by the mechanical properties of the CF-HEC biocomposite.

\section{Experimental}

Materials

Never-dried dissolving softwood pulp with a solid content of 15-16 wt\% (Domsjö Fabriker, Örnsköldsvik, Sweden) and with the chemical composition of $3.5 \mathrm{wt} \%$ hemicellulose, $0.4 \mathrm{wt} \%$ lignin and $0.5 \mathrm{wt} \%$ inorganics (TAPPI-T 222 standard) was used in this work. Hydroxyethyl cellulose (HEC) is a cellulose derivative and with an average molecular weight of $1,300,000$ and density of $0.6 \mathrm{~g} / \mathrm{mL}$ (at $25^{\circ} \mathrm{C}$ ) was purchased from Sigma Aldrich (Steinheim, Germany) and used to prepare the dissolved pulp and HEC mixtures for extrusion processing. The role of the HEC 
is to act as processing aid in the TSE process, it is a water soluble and also as a matrix polymer in the final composite. Deionized water was used in this investigation.

\section{Manufacturing process}

The manufacturing process starts with preparation of different CF-HEC aqueous dispersions with different $\mathrm{CF}$ and HEC proportions (Table 1) and with an aqueous concentration of $20 \mathrm{wt} \%$. The decided amounts of HEC and water were first mixed for $10 \mathrm{~min}$ at room temperature, and then the $\mathrm{CF}$ was added to the HEC-water dispersion and mixed using a mixer at $100 \mathrm{rpm}$ at room temperature for $30 \mathrm{~min}$. The different CF-HEC mixtures and their contents were collected on aluminum foil and dried in an oven at $50{ }^{\circ} \mathrm{C}$ for as long as the total solid content of the CFHEC medium reached $40-43 \mathrm{wt} \%$ for a further onestep TSE process.

The CF-HEC mixtures with a solid content of $>40$ wt $\%$ were extruded using a co-rotating twin-screw extruder (ZSK-18 MEGALab, Coperion W\&P, Germany) with a screw speed of $200 \mathrm{rpm}$. The processing temperature was set to $140{ }^{\circ} \mathrm{C}$ for all 7 processing zones and increased to $160{ }^{\circ} \mathrm{C}$ at the die in extrusion, the temperature was kept below the degradation temperature of HEC as it do not melt. The extrusion was made without the die tool, to be able to reduce the pressure at the end of the process and because the material is in a form of dry "powder" it can block the die. The residence time of CF-HEC in the extruder was around $90-95 \mathrm{~s}$. The low processing temperature was used to avoid degradation of the materials and to evaporate the water. The moisture content of the extrudate (CF-HEC powder) was measured to be around $5-8 \%$. The screw configuration, shown in Fig. 1, include shear as well as dispersive and distributive elements for the processing of CF-HEC mixtures. After the extrusion, the solid content of the CF-HEC increased 92-95 wt $\%$. The extruded materials EX-50CF, EX-65CF, and EX-80CF were diluted by adding extra HEC (dry mixing) to EX-20CF, EX$30 \mathrm{CF}$, and EX-40CF for further characterization. CFHEC biocomposites were then compression-molded using a laboratory press (LabEcon 300, Fontijne Press, Vlaardingen, Netherlands). The CF-HEC powder (after extrusion) forms some small flocs due to 5-8 wt $\%$ moisture content during the sample collection. The powder-floc materials were compression-molded to prepare the biocomposite sheet. The biocomposite powder-flocs were diluted with extra HEC (dry mixing) to enable pressing with the lower fiber content between aluminum plates and Teflon films.

The extruded CF-HEC (with 5-8\% moisture content) first weighted and loaded (uniformly distributed) on the teflon-covered plate frame $(0.2 \mathrm{~m}$ in $0.2 \mathrm{~m})$, then the pressure applied. The compression molding was done using a heating-cooling protocol under force of $50 \mathrm{kN}(1.25 \mathrm{MPa})$ at $145{ }^{\circ} \mathrm{C}$ for $2 \mathrm{~min}$, followed by increased force to $90 \mathrm{kN}(2.25 \mathrm{MPa})$ at $145{ }^{\circ} \mathrm{C}$ for $8 \mathrm{~min}$, and then $90 \mathrm{kN}(2.25 \mathrm{MPa})$ at $100{ }^{\circ} \mathrm{C}$ for

Table 1 The sample coding for the used material compositions

\begin{tabular}{|c|c|c|c|c|}
\hline Sample name & $\mathrm{CF}(\mathrm{wt} \%)$ & HEC (wt\%) & Pre-preparation method & Solid content before hot-pressing $(\mathrm{wt} \%)^{\mathrm{a}}$ \\
\hline $\mathrm{CF}$ & 100 & - & - & - \\
\hline HEC & 0 & 100 & - & $>95$ \\
\hline EX-50CF & 50 & 50 & Extruded & $>95$ \\
\hline EX-65CF & 65 & 35 & Extruded & $>95$ \\
\hline EX-80CF & 80 & 20 & Extruded & $>95$ \\
\hline $\mathrm{EX}-20 \mathrm{CF}^{\mathrm{b}}$ & 20 & 80 & Extruded-Diluted & $>95$ \\
\hline $\mathrm{EX}-30 \mathrm{CF}^{\mathrm{b}}$ & 30 & 70 & Extruded-Diluted & $>95$ \\
\hline $\mathrm{EX}-40 \mathrm{CF}^{\mathrm{b}}$ & 40 & 60 & Extruded-Diluted & $>95$ \\
\hline
\end{tabular}

The samples were used for different characterizations, e.g., morphology, rheology and tensile test. The values in parentheses are standard deviations

${ }^{\mathrm{a}}$ The HEC was used in powder form for the hot-pressed specimens

${ }^{\mathrm{b}}$ The EX-20CF, EX-30CF, and EX-40CF samples were prepared by diluting prior EX-50CF, EX-65CF, and EX-80CF, respectively 


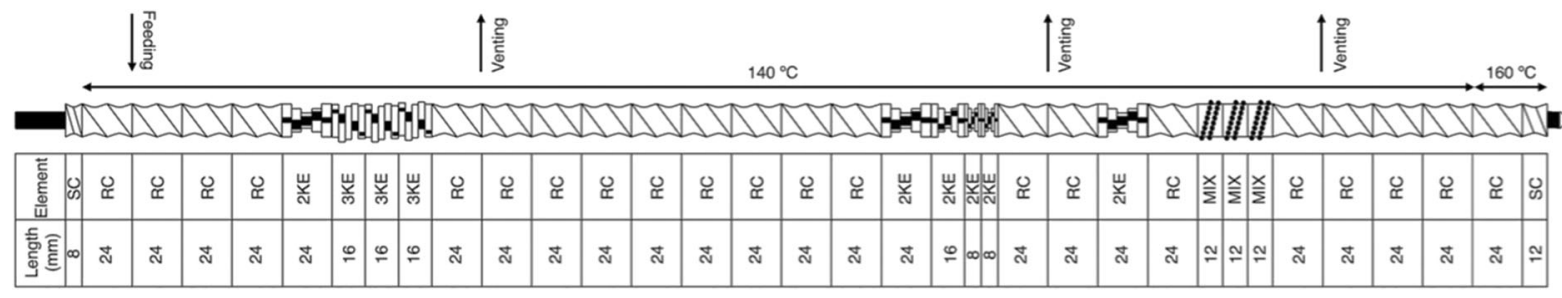

Fig. 1 The used twin-screw extruder set-up, showing the screw configuration with short conveying (SC) regular conveying (RC), double flights kneading (2KE), triple flights kneading

2 min, continued with $50 \mathrm{kN}(1.25 \mathrm{MPa})$ at $50{ }^{\circ} \mathrm{C}$ for $2 \mathrm{~min}$, and ultimately $50 \mathrm{kN}(1.25 \mathrm{MPa})$ at $25{ }^{\circ} \mathrm{C}$ for $1 \mathrm{~min}$ (Fig. 2). The temperature of the hot-pressed was optimized to avoid degradation and the biocomposite color was observed without any change. CF-HEC biocomposite films cut (for tensile test) using a Roland SRM-20 milling machine (USA) and the use of a $1 / 162 \mathrm{~mm}$ screw mill. The prepared specimens (cut by a milling machine) with a length of $30 \mathrm{~mm}$ and a width of $5 \mathrm{~mm}$ were used for tensile test measurements. The prepared specimens (for tensile test) were stored in testing conditions at the relative humidity (RH) of $50 \%$ at $23{ }^{\circ} \mathrm{C}$ for several days.

\section{Materials characterization}

Size reduction and morphology

Before the tube flow fractionation test and microscopy studies, the HEC was removed by washing the composite three times with warm water $\left(70{ }^{\circ} \mathrm{C}\right)$, followed with vacuum filtering using a filter paper with the pore size of $12-15 \mu \mathrm{m}$ (VWR, Leuven, Belgium). After washing, the waste water (all washing
(3KE), and tooth mixing (MIX) elements, temperature settings, and feeding and venting zones

steps) was collected and the removed HEC in waste water was calculated between $92-95 \%$. The extracted fibers were diluted to $0.3 \mathrm{wt} \%$ prior to the tube flow fractionation test and $0.1 \mathrm{wt} \%$ for the OM studies and $0.01 \mathrm{wt} \%$ for FE-SEM, and vacuum-filtered via a Nuclepore Track-Etch membrane with the pore size of $0.2 \mu \mathrm{m}$ (Whatman, UK).The size reduction of the fibers after the extrusion was studied using a tube flow fractionation test (Valmet Automation Oy, Kajaani, Finland) equipped with IMG image analyzer software. The fractionator is equipped with a high-speed CCD camera, and the fiber images were captured in a very small time-scale (millisecond) for image processing. This method is thoroughly explained in different works (Laitinen and Niinimäki 2014). The measurement data and the captured video (around 2000 images) were used to investigate the fiber length, width, and aspect ratio before and after the TSE.

The morphology of the fibers before and after processing was examined using optical microscopy (OM) (Leica MZ FL III, Leica Camera AG, Wetzlar, Germany) and field emission scanning electron microscope (FE-SEM) (ZEISS ULTRA plus FE-SEM, Carl Zeiss AG, Oberkochen, Germany). FE-SEM was used to study the microstructure of the CFs. The collected

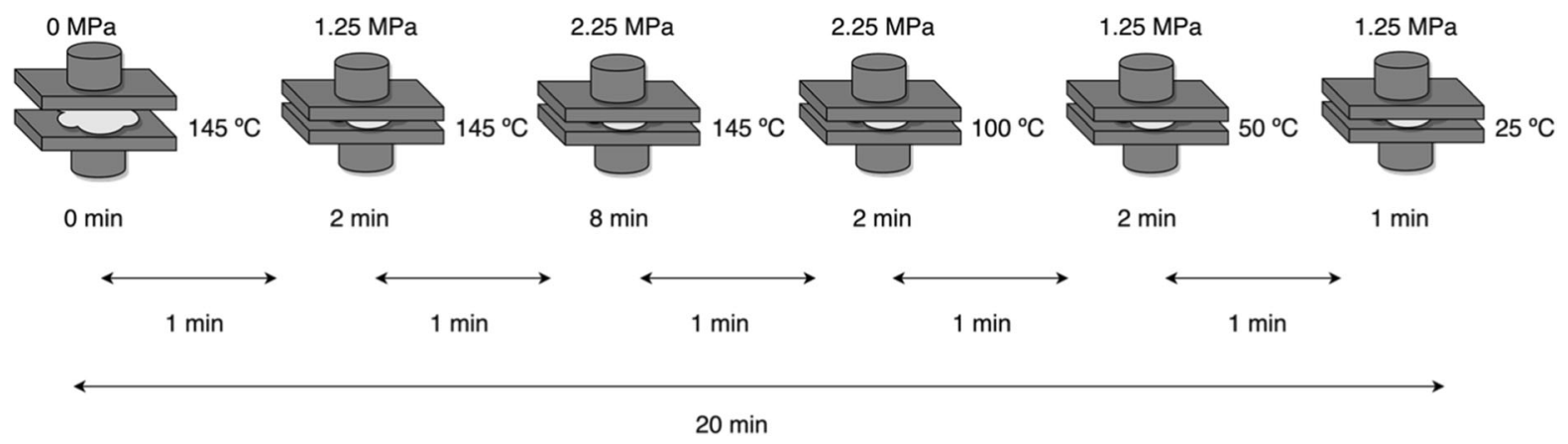

Fig. 2 The time-temperature-pressure protocol of CF-HEC biocomposite during hot-pressing 
fibers were immediately frozen in liquid nitrogen and freeze-dried for $24 \mathrm{~h}$. The samples were mounted on sample holders and platinum-coated before FE-SEM to avoid charging. The acceleration voltage of $5 \mathrm{kV}$ was applied in all FE-SEM observations.

\section{Rheology}

The rheological measurements of CF-HEC dispersions were done using a TA Instrument Discovery HR1 Hybride Rheometer (New Castle, DE, USA). Different aqueous samples were prepared with total solid contents of $2.5 \mathrm{wt} \%$ to compare the flow behavior of the bulk CF-HEC after the extrusion process to study how the flow properties of the materials change due to fiber content and the TSE process. The rheological behavior of the samples under the straincontrolled mode was measured by bob and cup accessories and with the diameters of $27.97 \mathrm{~mm}$ and $30.35 \mathrm{~mm}$, respectively. The steady state shear-viscosity tests were done to investigate the rheological properties of bulk CF-HEC at $25{ }^{\circ} \mathrm{C}$. The wall-slip effect of the test protocol for the used medium was also checked in three different gaps $(1.5,2$, and $2.5 \mathrm{~mm})$, and the shear-viscosity data were reproduced for all gaps, confirming the absence of the wall-slip effect as shown in the supplementary materials (Fig. A1). The reference gap distance of $1.5 \mathrm{~mm}$ was used for all experiments, and the gap slowly adjusted for $2 \mathrm{~min}$ to erase additional stress and equilibrate the temperature between sample and cup. Water evaporation was also controlled with use of a standard hood cover for all experiments. The rotational measurements were done under a controlled shear rate in a ramp-up and rampdown sequence of about $32 \mathrm{~min}$ (15 min for ramping up, $2 \mathrm{~min}$ at maximum shear rate, and finally $15 \mathrm{~min}$ for ramping down) to show the suspension stability at shear rates $0.1-1000 \mathrm{~s}^{-1}$ (within $25 \mathrm{~min}$ ) at $25^{\circ} \mathrm{C}$.

\section{Mechanical properties}

The tensile properties of the biocomposite materials were measured using a universal testing machine (Zwick, Ulm, Germany) equipped with a $200 \mathrm{~N}$ load cell, and all experiments were done following the DIN EN ISO 527-1 film standard. The compression-molded biocomposite specimens with the width $5 \mathrm{~mm}$, length $30 \mathrm{~mm}$, and thickness $350-540 \mu \mathrm{m}$ were tested. The thickness of each specimen was determined as an average from three random locations on the specimen, using a micrometer Rhopoint instrument (Bexhill-onsea, UK). The specimens were stored in $50 \% \mathrm{RH}$ at $23{ }^{\circ} \mathrm{C}$ in the measurement environment. The gauge length was set to $15 \mathrm{~mm}$; the strain rate of $1 \% / \mathrm{min}$ and the samples were preloaded to $1 \mathrm{~N}$. The results are presented as an average of six measurements.

\section{Results and discussion}

\section{Fibrillation}

The effect of one-step TSE process on cellulose fiber size reduction was studied using tube flow fractionation test and the results are shown in Fig. 3. Micrographs of 5 different fractions of the EX-50CF, EX$65 \mathrm{CF}$, and EX-80CF after the TSE are compared with non-extruded $\mathrm{CF}$; all the studied fiber dispersions had same cellulose concentration, $0.3 \mathrm{wt} \%$. The micrographs clearly show that one-step TSE resulted in smaller fiber size at the largest fraction (Fraction 1), which is $21-27 \mu \mathrm{m}$. The large fibers were almost nonexistent after the extrusion in the third and fourth fractions, and the small fibers are increasing. Furthermore, the fractional numerical data of the different fractions are listed in Table 2.

According to the fractionation test, the proper defibrillation must result in the lowest amount (\%) of thick fibers and the highest amount (\%) of thin fibers. Therefore, with the proper extrusion and defibrillation, the first two fractions decreased, and the last two fractions increased. According to the fractional data, the one-step TSE process resulted in fibrillation, to significant reduction of fiber size and increment of the thinner fibers.

The results are also shown in in Table 2, Fraction 1 contained the largest fibers, thus confirming fiber breakage of the CF after TSE, as the number of fibers decreased from $76 \%$ original $\mathrm{CF}$ to $3 \%$ for the extruded EX-80CF. It is also possible to see a slight effect with other solid contents (EX-50CF and EX65CF), with around 9\% fibers in Fraction 1. The amount of fibers in Fraction 2 decreased from $10 \%$ (for the original $\mathrm{CF}$ ) to $25 \%, 8.6 \%$, and $6.1 \%$ for the extruded samples EX-50CF, EX-65CF, and EX-80CF, respectively. The amount (\%) of the fibers (13-21 $\mu \mathrm{m})$ in Fraction 2 was also decreased from 25\% (EX-50CF) to $6.1 \%(\mathrm{EX}-80 \mathrm{CF})$ with the increment of $\mathrm{CF}$ 


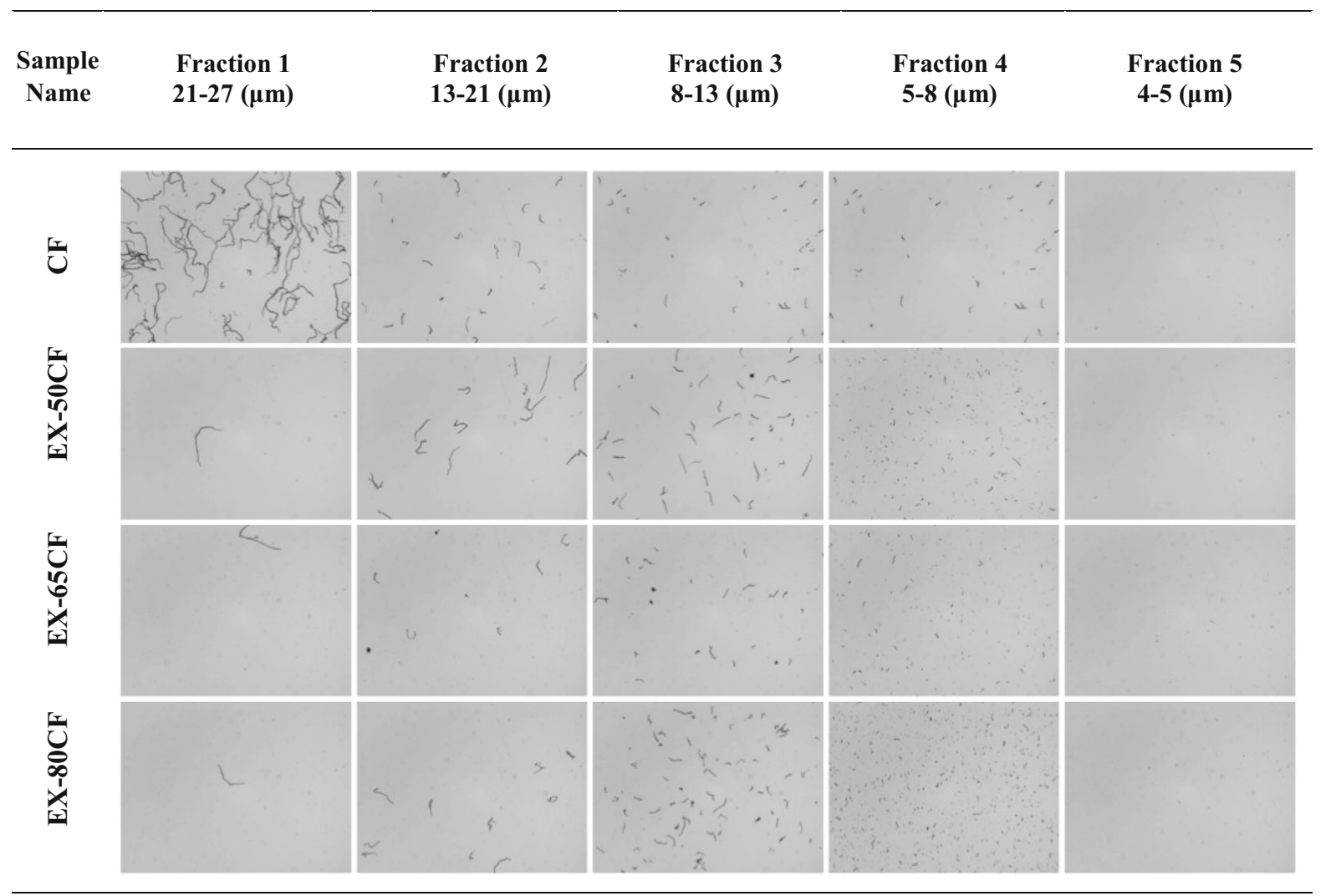

Fig. 3 The micrographs of tube flow fractionation test of original $\mathrm{CF}$ and extruded $\mathrm{CF}$ in water dispersion $0.3 \mathrm{wt} \%$ with 5 different fiber width fractions. It was seen that Fraction 1, the largest fiber length, was dramatically reduced after the TSE step for all material combinations when compared with original $\mathrm{CF}$. It is also clearly visible that Fractions 3 and 4 increased and were the highest for the EX-80CF

Table 2 Fiber width data reported by tube flow fractionation test and via image processing for the original $\mathrm{CF}$ and extruded $\mathrm{CF}$ samples

\begin{tabular}{|c|c|c|c|c|c|c|c|c|c|c|}
\hline \multirow{2}{*}{$\begin{array}{l}\text { Sample } \\
\text { name }\end{array}$} & \multicolumn{2}{|c|}{ Fraction 1} & \multicolumn{2}{|l|}{ Fraction 2} & \multicolumn{2}{|l|}{ Fraction 3} & \multicolumn{2}{|l|}{ Fraction 4} & \multicolumn{2}{|l|}{ Fraction 5} \\
\hline & $\begin{array}{l}\text { Fiber } \\
\text { width }^{\mathrm{a}} \\
(\mu \mathrm{m})\end{array}$ & $(\%)$ & $\begin{array}{l}\text { Fiber width } \\
(\mu \mathrm{m})\end{array}$ & $(\%)$ & $\begin{array}{l}\text { Fiber width }{ }^{\mathrm{a}} \\
(\mu \mathrm{m})\end{array}$ & $(\%)$ & $\begin{array}{l}\text { Fiber width } \\
(\mu \mathrm{m})\end{array}$ & $(\%)$ & $\begin{array}{l}\text { Fiber width } \\
(\mu \mathrm{m})\end{array}$ & $(\%)$ \\
\hline $\mathrm{CF}$ & 26.9 & 76.1 & 14.6 & 10.6 & 8.2 & 8.8 & 5.6 & 2.5 & 4.3 & 2.1 \\
\hline EX-50CF & 22.0 & 9.3 & 18.8 & 25.0 & 11.5 & 54.0 & 6.7 & 7.8 & 4.9 & 3.9 \\
\hline EX-65CF & 21.5 & 9.0 & 18.2 & 8.6 & 13.6 & 52.5 & 7.9 & 20.9 & 5.5 & 8.9 \\
\hline EX-80CF & 21.1 & 2.6 & 20.9 & 6.1 & 13.2 & 63.0 & 7.1 & 25.1 & 5.3 & 3.3 \\
\hline
\end{tabular}

The HEC was removed from the CF-HEC biocomposite before testing, and all materials had same cellulose concentration 0.3 wt $\%$ within testing

${ }^{\mathrm{a}}$ The average fiber width was calculated by image processing 
proportion in CF-HEC biocomposite. Fraction 3 represents the middle fiber width $(8-13 \mu \mathrm{m})$. According to the fractional data $(\%)$, more than half of the extruded fibers were observed in the third fraction. Therefore, the width of the original CF decreased more than $50 \%$ after extrusion, confirming the width reduction of $50 \%$ of non-extruded CF. In Fraction 4, the amount $(\%)$ of fine fibers (width: $5-8 \mu \mathrm{m}$ ) are presented and shown; in comparison to the original $\mathrm{CF}$, the amount of Fraction 4 increased after extrusion, especially in the cases of EX-65CF and EX-80CF. Fraction 5 represents the finest fibers during the tube flow fraction test, which is low for all samples.

As the recorded data via high speed CCD camera has some limitations in width range (lower than $5 \mu \mathrm{m}$ ), other characterization methods can be more reliable in analyzing the fine fibers in the fifth fraction. The ISO length of the fiber is important data, which can be collected from the tube flow fraction test. Therefore, the length reduction and the aspect ratio of the CF can also be calculated (Table 3). In this study, the length reductions of $50 \%, 70 \%$, and $69 \%$ were calculated for EX-50CF, EX-65CF, and EX-80CF, respectively. It has been reported that extrusion can result in fiber breakage (Le Baillif and Oksman 2009; Hietala and Oksman 2018), and the results presented in Table 3 also show the length reduction after extrusion. The aspect ratios were also decreased from 56 for the neat CF to 32-47 for the extruded CF. In brief, the extruded $\mathrm{CF}$ with the lower $\mathrm{CF}$ proportion and higher amount of HEC (EX-50CF) has a greater aspect ratio with a lower amount $(\%)$ of middle and fine fractions. Unlike the extruded CF with the higher fiber proportion (EX$80 \mathrm{CF}$ ) has a greater amount (\%) of middle and fine fractions, whereas its aspect ratio was reduced up to
32. The shear forces are different within the used screw profile, and we expect that the kneading elements are resulting in highest shear (friction) and resulting in fibrillation but also to fiber breakage. We can also expect that the increased HEC content decreases the direct shear on the cellulose possible due to the lubricating effect and thus resulting in less breakage.

\section{Morphology}

The optical microscopy of the CF-HEC mixtures with different $\mathrm{CF}$ concentrations (after HEC removal) was done to investigate the size alteration of the cellulose fiber after having been processed by one-step TSE (Fig. 4). The OM for the neat CF was also done (as shown in Fig. 4a) to compare with extruded CF micrographs. These observations confirmed the clear size alteration (in both width and length) of the CFs with the one-step TSE process, in agreement with other investigations (Ho et al. 2015; Rol et al. 2017).

However, with the lower CF proportion (EX-50CF) having higher HEC content, the size reduction was minimized in comparison with the higher CF proportion (EX-65CF and EX-80CF). Therefore, the extruded biocomposite with the lower CF proportion showed less reduction in the width and length of the fibers (Fig. 4b). With the increment of the CF proportions from $50 \%$ (EX-50CF) to $65 \%$ and $80 \%$ (EX-65CF and EX-80CF), the size reduction was observed in Fig. $4 c$ and d, respectively. The size reduction of the extruded $\mathrm{CF}$ was also in agreement with tube flow fraction measurements, as presented in Table 3. Moreover, the comparison of micrographs (Fig. 4) and tube flow fraction test data (Table 3)

Table 3 Dimensions and aspect ratio of neat CF and extruded CF, which were separated from CF-HEC biocomposite

\begin{tabular}{|c|c|c|c|c|c|}
\hline Sample name & Fiber length (1) $(\mathrm{mm})^{\mathrm{a}}$ & Fiber width $(\mathrm{d})(\mu \mathrm{m})^{\mathrm{b}}$ & Fiber width $(\mathrm{d})(\mu \mathrm{m})^{\mathrm{c}}$ & Aspect ratio (1/d) & Length reduction $(\%)$ \\
\hline $\mathrm{CF}$ & $1.30(0.02)$ & $26.87(2.36)$ & 22.92 & 56 & - \\
\hline EX-50CF & $0.64(0.01)$ & $20.93(0.19)$ & 13.65 & 47 & 50 \\
\hline EX-65CF & $0.39(0.01)$ & $21.68(0.42)$ & 12.78 & 30 & 70 \\
\hline EX-80CF & $0.40(0.01)$ & $21.47(0.61)$ & 12.22 & 32 & 69 \\
\hline
\end{tabular}

The values in parentheses are standard deviations

${ }^{\mathrm{a}}$ The ISO fiber length

${ }^{\mathrm{b}}$ Fractionator data processing (weight system)

${ }^{\mathrm{c}}$ Calculated by image processing 


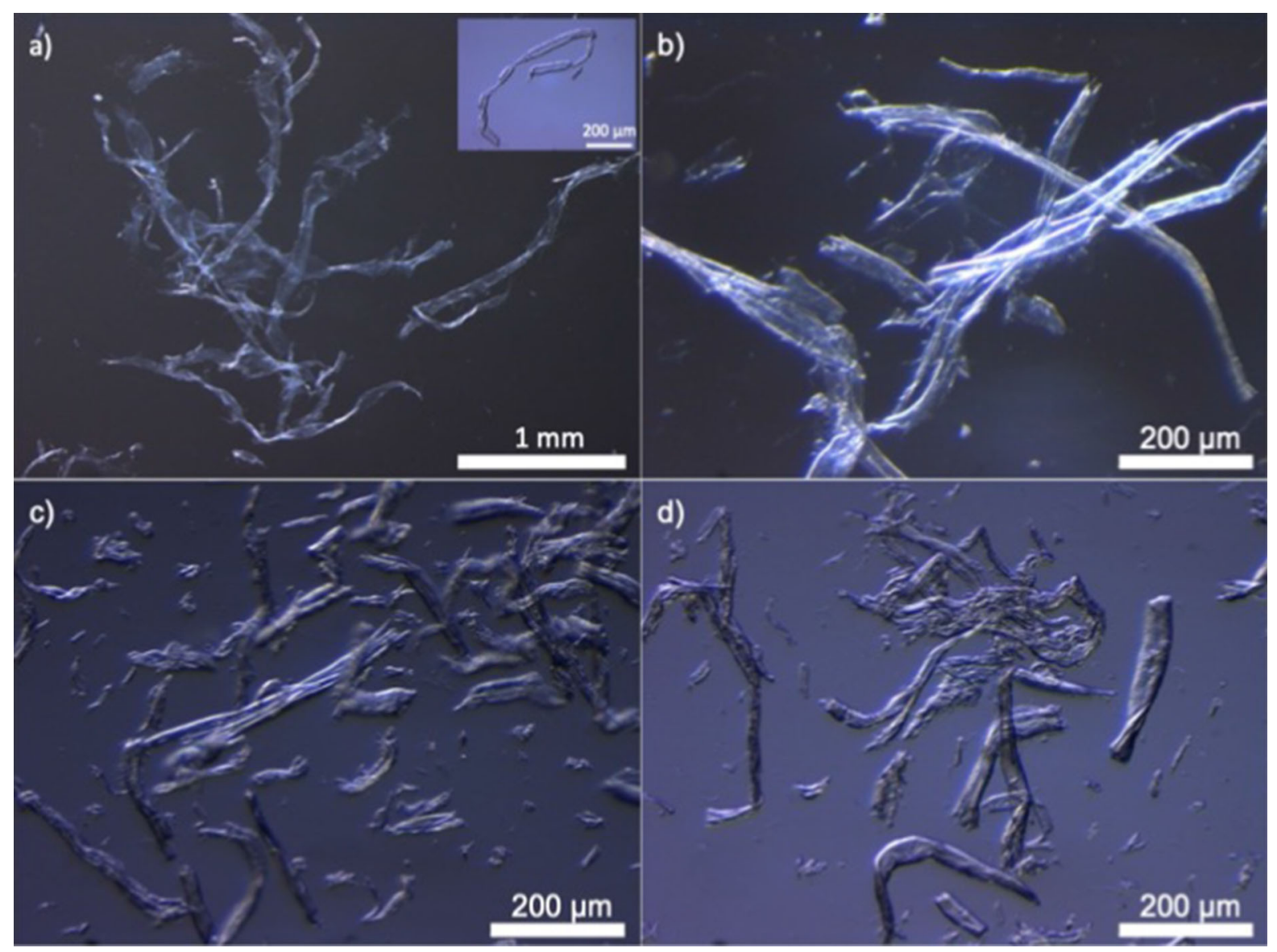

Fig. 4 Optical microscopy of original CF and TSE processed CF; a original CF, b EX-50CF, c EX-65CF and d EX-80CF. Inset of a image obtained at higher magnification

confirmed the greater length reduction and smaller width reduction with the increment of CF proportion.

HEC was removed by washing but it was recognized that some HEC remained in the fiber surfaces and this can affect the width measurements from both micrographs and fractionator tests.

The FE-SEM images of neat CF and extruded CF with different fiber concentrations are shown in Fig. 5. The FE-SEM of neat CF (Fig. 5a) show cellulose fibers with typical morphology. The Fig. 5b-d, show extruded $\mathrm{CF}$ and size reduction especially on the materials with higher $\mathrm{CF}$ concentrations (Fig. 5c and d) is visible. It is possible that there is some HEC adsorbed on the cellulose fiber surfaces and is not completely removed by washing, that is visible especially in the sample with highest HEC concentration (Fig. 5b). Therefore, the width of extruded fibers, which were reported by image processing, can be more reliable than the fractionator measurements (Table 3).

The one-step TSE of CF with higher fiber proportion resulted in a finer fibril and a greater morphological change. As the higher CF proportion (EX-80CF) showed greater morphological change, the FE-SEM of this sample was also observed for each of the fractions of the fractionator test, as shown in the supplementary materials (Fig. A2). In this regard, the FE-SEM images also confirmed the width reductions for each fraction. The microstructural observations showed that the onestep TSE mixtures with different CF proportions represented different fiber size ranges, which contained a greater amount of thicker fibers for EX-50CF, middle fiber range for EX-65CF, and greater amount of thinner fibers for EX-80CF. Moreover, the fibrillation of CFs was done by using one-step TSE for all samples with different $\mathrm{CF}$ amounts, and it seems that 

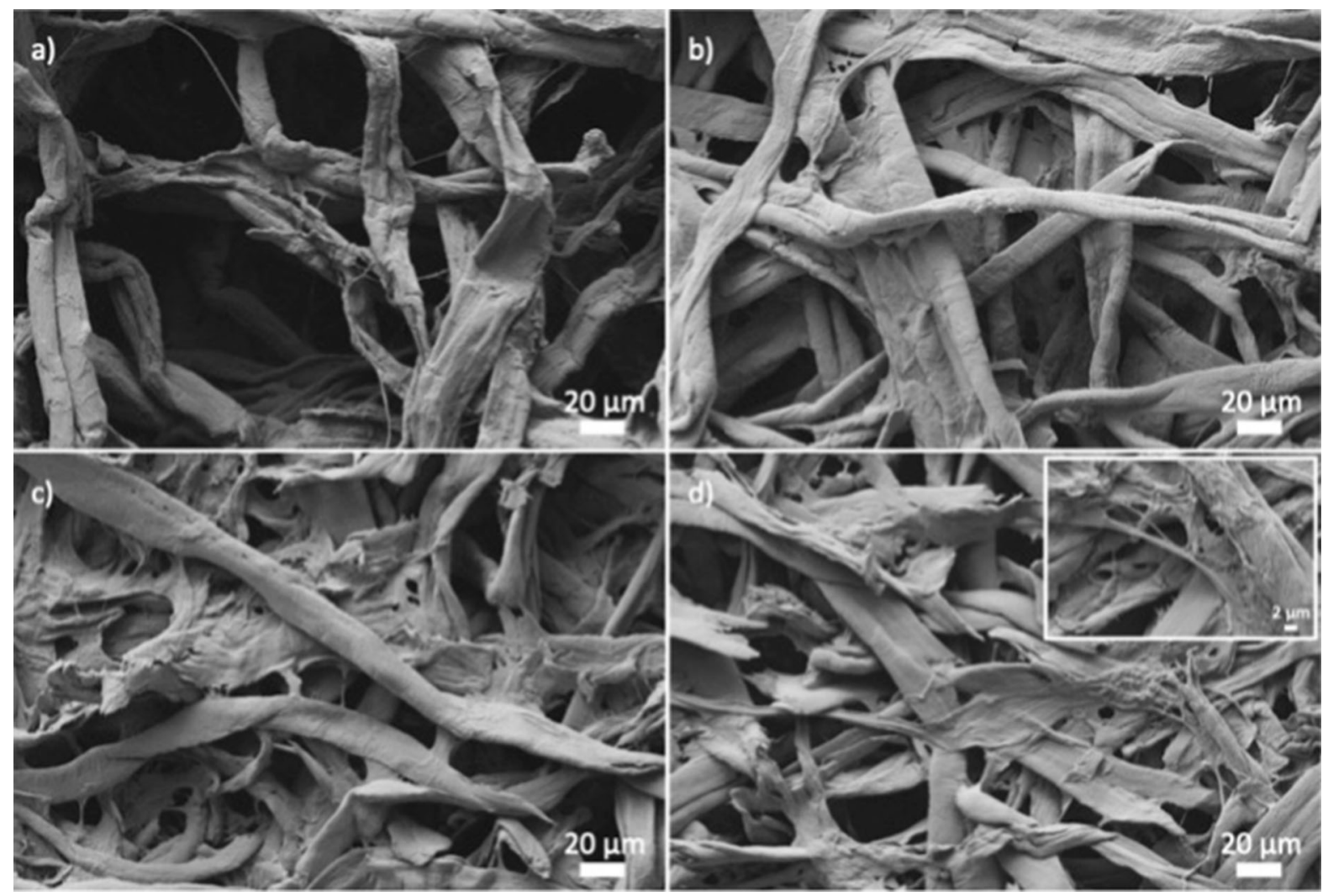

Fig. 5 The FE-SEM images of original CF and extruded CF by one-step TSE; a original CF, b EX-50CF, c EX-65CF and d EX-80CF. Inset of $\mathbf{d}$ image obtained at higher magnification

the higher fiber content in relation to HEC is leading to greater changes in fiber dimensions within the onestep TSE process.

\section{Viscosity}

The rheological measurements of the suspensions can be applied as suitable complementary data (along with other methods, e.g., FE-SEM and OM) to elucidate both the flow properties and morphological changes after the extrusion process. As the rheological measurements are provided with a large amount of material (in comparison with other methods using small amounts of material), the flow properties and morphological changes can be firmly reflected in the behavior of the bulk sample, resulting in more reliable interpretations. In this respect, the rheological behaviors of the CF-HEC water dispersions with different CF concentrations were measured after one-step TSE, as shown in Fig. 6. The rheological behavior of

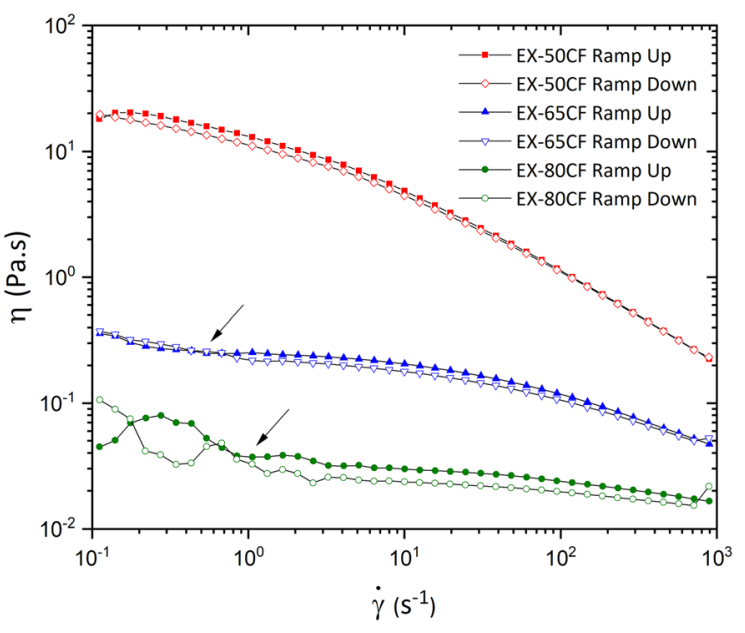

Fig. 6 Shear rate versus viscosity plots of CF-HEC suspensions (2.5 wt \%) with different fiber proportion of 50, 65 and $80 \%$ (Table 1) at $25^{\circ} \mathrm{C}$ after one-step TSE. The solid symbols indicate the ascending shear rate and the open symbols indicate the descending shear rate 
extruded samples was also measured before extrusion, as shown in the supplementary materials (Fig. A3).

The shear-viscosity data was first done in a ramp-up (ascending shear rate) mode and then in a ramp-down mode (descending shear rate). The shear-viscosity plots showed shear thinning behavior for all samples after extrusion. The rotational shear rate data (after one-step TSE) confirmed that the overall shearviscosity trends (Fig. 6) of CF-HEC suspension were clearly decreased, with increments of fiber proportion from $50 \%$ (EX-50CF) to $80 \%$ (EX-80CF). The decrement of these trends can be explained by fiber size reduction. The rotational tests also showed some hysteresis between the ramp-up and ramp-down data. The mentioned hysteresis between ascending and descending shear data was increased with the increment of the fiber proportion (Fig. 6).

In general, the instability of the dispersions can be indicated by hysteresis between the ramp-up and ramp-down data or two-phase behavior (Bröckel et al. 2013). Therefore, the two-phase behavior of the suspension might be increased by aggregation or flocculation of the multi-scale fibers within the rotational shear rate. Herein, the size reduction will be maximized with increments of fiber content within the TSE process and the plots showed a slight plateau at the shear rate of 0.5 and $1 \mathrm{~s}^{-1}$ for EX-65CF and EX$80 \mathrm{CF}$, respectively. The mentioned plateau (shown with arrows in Fig. 6) represents the fingerprint of fibrillation of the fibers in conventional rotational rheometry (Taheri and Samyn, 2016). The comparison of rheological data of the extruded samples (Fig. 6) and the non-extruded samples (Fig. A3) confirmed the effect of the TSE process and fiber content. However, the overall shear-viscosity trends of the extruded samples were abruptly decreased by the increments of the fiber proportion, which is related to the size reduction of the extruded fiber, confirming the tube flow fraction data and micrograph observations. Moreover, the comparison of rheological data of the extruded samples (Fig. 6) and the non-extruded samples (Fig. A3) showed very close viscosity (20-24 Pa s) at low shear rate for the samples with the equal fiber-matrix proportion (1:1). Therefore, any sort of degradation of CF-HEC biocomposite within extrusion can be tracked from rheological data (suspension rheology) before and after extrusion. In this regard, the viscosity drops for EX-65CF and EX-80CF are related to fiber size reduction.

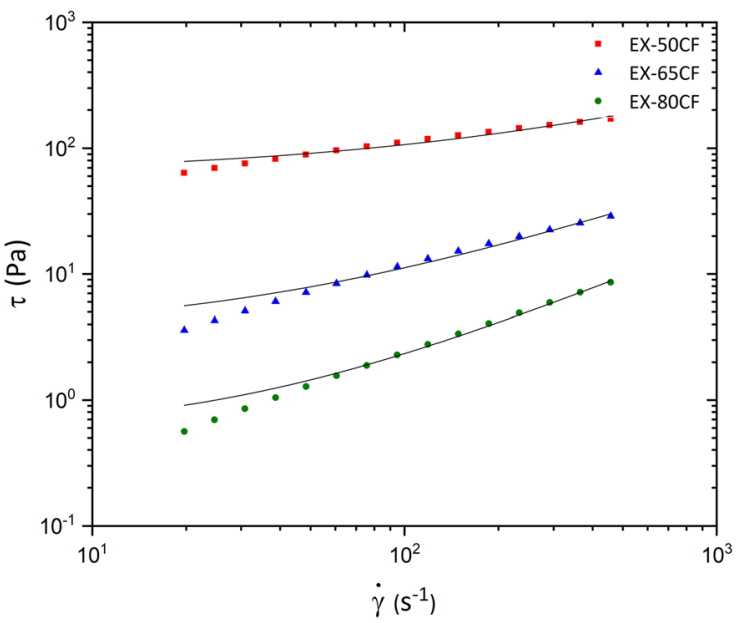

Fig. 7 Shear rate versus stress plots of CF-HEC suspensions $(2.5 \mathrm{wt} \%)$ with different fiber proportion of 50,65 and $80 \%$ at $25{ }^{\circ} \mathrm{C}$ after one-step TSE. The apparent yield stress $\left(\tau_{0}\right)$ of the samples was determined by fitting (line) of the HerschelBulkley model

The yield stress of suspensions is an important rheological parameter that is related to flow properties or the onset of the flow. Based on the conventional rotational rheometry, the shear rate and shear stress data can be used to define the yield stress of the CFHEC suspensions (Fig. 7). The flow behavior of the fluid is related to the yield stress in a complicated nonlinear curve that can be determined by the HerschelBulkley model. The Herschel-Bulkley model is shown in Eq. (1):

$\tau=\tau_{0}+k(\dot{\gamma})^{n}$

where the parameters $k, n$, and $\tau_{0}$ are related to consistency, flow index and the yield stress.

The Herschel-Bulkley model cannot be used for the Newtonian plateau at intermediate shear rates (Karppinen et al. 2011). Therefore, the Herschel-Bulkley fits as shown in Fig. 7, which were done only at shear rates above the transition region between 20 and $500 \mathrm{~s}^{-1}$.

According to the Herschel-Bulkley fits (Fig. 7), the apparent yield stresses of the CF-HEC suspensions after extrusion (as shown in Table 4) were decreased, with an increment of the fiber proportion from 63.77 to $0.56 \mathrm{~Pa}$, which can occur due to lesser binding or entanglement between the fibers. The reduction of the yield stress for the extruded CF-HEC (with different $\mathrm{CF}$ proportions) confirmed the likely increment of the shear forces (friction) between fibers with higher $\mathrm{CF}$ 
Table 4 The yield stress and different parameters of extruded CF-HEC samples with the use of Herschel-Bulkley model

\begin{tabular}{lllll}
\hline Sample name & Yield stress $(\mathrm{Pa})$ & $k$ & $n$ & $R^{2}$ \\
\hline EX-50CF & 63.77 & $2.14(0.58)$ & $0.65(0.04)$ & 0.97 \\
EX-65CF & 3.57 & $0.17(0.04)$ & $0.81(0.04)$ & 0.98 \\
EX-80CF & 0.56 & $0.01(0.01)$ & $1.02(0.02)$ & 0.98 \\
\hline
\end{tabular}

The values in parentheses are related to the fitting errors (Fig. 7)

proportions (EX-80CF). The extruded EX-50CF showed the highest yield stresses among all samples, which is related to the lower fibrillation of fibers and higher amounts of the HEC matrix. According to these results, it could be possible to extrude the high fiber content CF-HEC material and then dilute the composite by adding extra HEC for further tensile characterizations.

\section{Mechanical properties}

The mechanical properties of the CF-HEC biocomposite with different fiber contents are shown in Fig. 8. Comparison of the mechanical properties of neat HEC and one-step extruded CF-HEC composites (EX50CF, EX-65CF, and EX-80CF) confirmed that the E-modulus (Fig. 8a) of extruded samples was increased with the increment of the fiber contents. The mentioned increment in E-modulus confirmed the higher friction between fibers and greater fibrillation of the fibers with higher fiber proportions. The tensile strength of EX-50CF in comparison with neat HEC was slightly decreased from 9.83 to $8.97 \mathrm{MPa}$, which can be related to fiber aggregation and lower fibrillation (Fig. 8b). The tensile strength of EX-65CF had a slight improvement and greater increment for EX$80 \mathrm{CF}$, which is either related to better mixing or fiber size reduction. However, the tensile strength of the CF-HEC composite improved with a greater amount of $\mathrm{CF}$ content than $50 \%$. In this regard, the tensile strength of CF-HEC biocomposite increased from 11.24 to $15.89 \mathrm{MPa}$ for EX-65CF and EX-CF80, respectively. The strain at the break (Fig. 8c) for the extruded samples was increased with the increment of the CF proportion. According to the tensile data, the strain at break of the neat HEC showed greater values in comparison with extruded CF-HEC samples. Therefore, the strain at the break seems to be more sensitive to the CF-HEC interfaces, fibers' interactions, fibers' entanglements, and fiber size reduction. As the number of hydrogen bonds between the $-\mathrm{OH}$ groups increases with the increment of the fibrillation, the E-modulus and tensile strength can be expected to increase. The tensile data of this work is in agreement with a report by Ho et al. (2015). However, the strain at break has a lower value in comparison with neat HEC.

As mentioned above, the strain at break can be related to the interaction between the matrix and fibers. Therefore, the extruded samples (EX-50P, EX65P, and EX-80P) were diluted by extra HEC (EX20CF, EX-30CF, and EX-40CF) to observe whether the strain at break will be improved. Interestingly, the E-modulus of all diluted samples improved in comparison with one-step extruded samples. The greatest E-modulus improvement was related to EX-40CF, which improved from 1.18 to $1.58 \mathrm{GPa}$ when adding more HEC to the extruded EX-80CF sample. As the CF-HEC biocomposite was primarily extruded with HEC matrix (EX-80CF), adding extra HEC cannot cause incompatibility or mixing issues. Therefore, the increase mentioned above in E-modulus can occur due to greater binding (gluing) or interface between the fiber particles and HEC matrix.

Adding more HEC (to the EX-65CF sample) to prepare the EX-30CF sample showed greater E-modulus than EX-50CF, EX-65CF, and EX-80CF. However, the E-modulus of EX-20CF and EX-30CF showed a close value around 1.28-1.48 GPa, but the EX-20CF sample showed a higher E-modulus in comparison with EX-30CF. In brief, the extruded CFHEC composite with extra HEC showed the maximum E-modulus of $1.58 \mathrm{GPa}$ for $\mathrm{EX}-40 \mathrm{CF}$, which was reduced to $1.28 \mathrm{GPa}$ for $\mathrm{EX}-30 \mathrm{CF}$ and then increased to $1.46 \mathrm{GPa}$ for EX-20CF. The main reason for this fluctuation can be expressed by a higher aspect ratio 
(a)
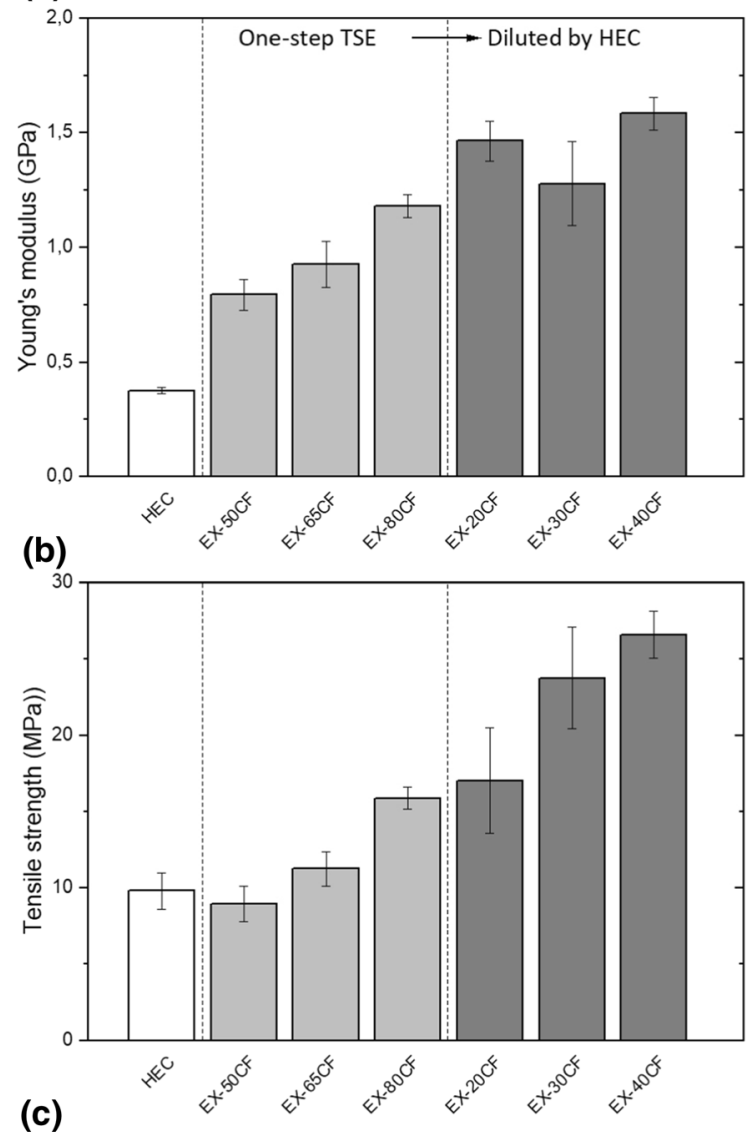

(c)

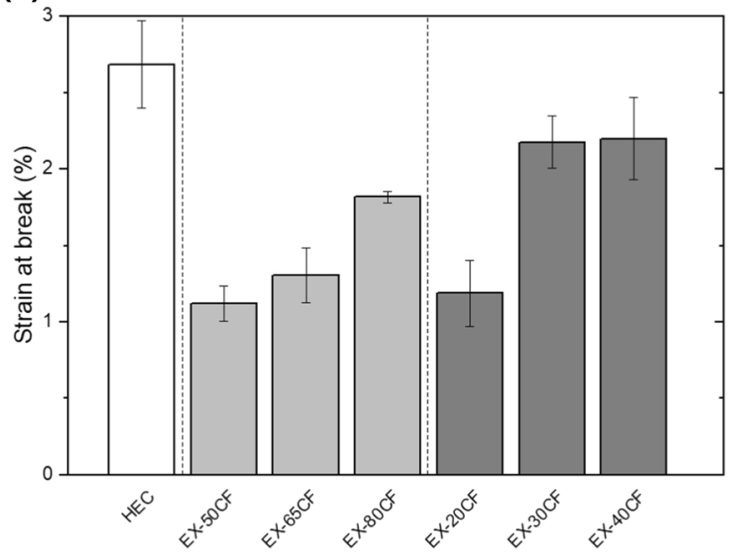

Fig. 8 Tensile behavior of neat HEC, extruded CF-HEC, and extruded-diluted CF-HEC biocomposites with different $\mathrm{CF}$ proportions; a E-modulus, b tensile strength, and $\mathbf{c}$ strain at break. The EX-20CF, EX-30CF, and EX-40CF samples were prepared by diluting prior EX-50CF, EX-65CF, and EX-80CF, respectively

(Table 3) of the fiber in the EX-20CF sample, which was prepared by adding extra HEC to EX-50C.
According to Fig. 8b, the CF-HEC with extra HEC (EX-40CF) showed the maximum tensile strength of 26.57 MPa in comparison with neat HEC matrix and all extruded samples. Therefore, the higher fiber content extrusion (EX-80CF) led to higher size reduction, and adding extra HEC led to better interaction between fibrillated fibers and matrix. Unlike the E-modulus data, the tensile strength of EX-30CF is greater than EX-20CF, which can be related to the aspect ratio of the fiber. The presented tensile data confirms that the E-modulus and tensile strength (excluding EX-40CF and EX-80CF) of the samples were more related to greater fibrillation. Unlikely, the strain at the break was more affected by the interaction between fibers and matrix. In brief, this approach (onestep TSE, then dilution) effectively improved the E-modulus, the tensile strength, and the strain at break.

\section{Conclusions}

The aim of this work was to study defibrillation of cellulose fibers in one-step TSE together with a hydroxyethylene cellulose (HEC) and form biocomposite. According to the results, the one-step TSE process reduced the size of the CFs, and the higher $\mathrm{CF}$ content resulted in greater fibrillation probably due to the higher shear forces on the fibers during the TSE.

The tube flow fraction test showed the partial fibrillation of the extruded CFs and $80 \mathrm{wt} \% \mathrm{CF}$ content had highest degree of fibrillation. The size reduction was also confirmed by optical microscopy and electron microscopy studies. It was also seen that some HEC still reminded on the fiber surfaces after the washing step which might slightly affect the measured fibers dimensions. Based on the rheological behavior of CF-HEC water dispersions, the yield stress of the dispersion decreased with the increment of the $\mathrm{CF}$ proportion, confirming the size reduction of the fibers.

Mechanical properties were measured on compression molded biocomposites with higher HEC concentrations and the results showed that E-modulus and tensile strength of the CF-HEC biocomposite improvement, confirming that different fiber contents and only one-step TSE affects the morphology of the fiber and, ultimately, the mechanical properties of the CF-HEC biocomposite. 
Acknowledgments Open access funding provided by Luleå University of Technology. Business Finland (formerly The Finnish Funding Agency for Technology and Innovation, TEKES) is acknowledged for their financial support (Grant No. 1841/31/2014). The authors would like to thank Mrs. Elisa Wirkkala, Mr. Jarno Karvonen, and Mr. Jani Österlund for their help at the Fibre and Particle Engineering laboratory. Domsjö Fabriker in Örnsköldsvik Sweden is also acknowledged for providing the cellulose material.

\section{Complaince with ethical standards}

Conflict of interest The authors declare that they have no conflict of interests.

Open Access This article is licensed under a Creative Commons Attribution 4.0 International License, which permits use, sharing, adaptation, distribution and reproduction in any medium or format, as long as you give appropriate credit to the original author(s) and the source, provide a link to the Creative Commons licence, and indicate if changes were made. The images or other third party material in this article are included in the article's Creative Commons licence, unless indicated otherwise in a credit line to the material. If material is not included in the article's Creative Commons licence and your intended use is not permitted by statutory regulation or exceeds the permitted use, you will need to obtain permission directly from the copyright holder. To view a copy of this licence, visit http://creativecommons.org/licenses/by/4.0/.

\section{References}

Berglund LA, Peijs T (2010) Cellulose biocomposites-from bulk moldings to nanostructured systems. MRS Bull 35:201-207

Bröckel U, Meier W, Wagner G (2013) Product design and engineering: formulation of gels and pastes. Wiley, Heidelberg

Cobut A, Sehaqui H, Berglund LA (2014) Cellulose nanocomposites by melt compounding of TEMPO-treated wood fibers in thermoplastic starch matrix. BioResources 9:3276-3289

Foster EJ, Moon RJ, Agarwal UP, Bortner MJ, Bras J et al (2018) Current characterization methods for cellulose nanomaterials. ChemSocRev 47:2609-2679

Fox SC, Li B, Xu D, Edgar KJ (2011) Regioselective esterification and etherification of cellulose: a review. Biomacromolecules 12:1956-1972

Haque MM, Herrera N, Geng S, Oksman K (2018) Melt compounded nanocomposites with semi-interpenetrated network structure based on natural rubber, polyethylene, and carrot nanofibers. J Appl Polym Sci 135:45961

Hietala M, Niinimäki OK (2011) Processing of wood chipplastic composites: effect on wood particle size, microstructure and mechanical properties. Plast Rubber Compos 40:49-56
Hietala M, Oksman K (2018) Pelletized cellulose fibres used in twin-screw extrusion for biocomposite manufacturing: fibre breakage and dispersion. Compos A 109:538-545

Hietala M, Samuelsson E, Niinimäki J, Oksman K (2011a) The effect of pre-softened wood chips on wood fibre aspect ratio and mechanical properties of wood-polymer composites. Compos A 42:2110-2116

Hietala M, Niinimäki J, Oksman K (2011b) The use of twinscrew extrusion in processing of wood. The effect of processing parameter and pretreatment. BioResources 6:4615-4625

Hietala M, Mathew AP, Oksman K (2013) Bionanocomposites of thermoplastic starch and cellulose nanofibers manufactured using twin-screw extrusion. Eur Polym J 49:950-956

Hietala M, Rollo P, Kekäläinen K, Oksman K (2014) Extrusion processing of green biocomposites: compounding, fibrillation efficiency and fiber dispersion. J Appl Polym Sci 131:39981

Hooshmand S, Aitomäki Y, Berglund L, Mathew AP, Oksman K (2017) Enhanced alignment and mechanical properties through the use of hydroxyethyl cellulose in solvent-free native cellulose spun filaments. Compos Sci Technol 150:79-86

Ho TTT, Zimmermann T, Caseri W (2011) Preparation and characterization of cationic nanofibrillated cellulose from etherification and high shear disintegration processes. Cellulose 18:1391-1406

Ho TTT, Abe K, Zimmermann T, Yano H (2015) Nanofibrillation of pulp fibers by twin-screw extrusion. Cellulose 22:421-433

Iwamoto S, Nakagaito AN, Yano H (2007) Nano-fibrillation of pulp fibers for the processing of transparent nanocomposites. Appl Phys A 89:461-466

Josset S, Orsolini P, Siqueira G, Tejado A, Tingaut P, Zimmermann T (2014) Energy consumption of the nanofibrillation of bleached pulp, wheat straw and recycled newspaper through a grinding process. Nord Pulp Pap Res J 1:167-175

Kamel S, Ali N, Jahangir K, Shah SM, El-Gendy AA (2008) Pharmaceutical significance of cellulose: a review. Express Polym Lett 2:758-778

Karppinen A, Vesterinen AH, Saarinen T, Pietikäinen P, Seppälä J (2011) Effect of cationic polymethacrylates on the rheology and flocculation of microfibrillated cellulose. Cellulose 18:1381-1390

Laitinen O, Niinimäki J (2014) Fractional study of the microfibrillated cellulose. Tappi J 13:49-55

Lahtinen P, Liukkonen S, Pere J, Sneck A, Kangas H (2014) A comparative study of fibrillated fibers from different mechanical and chemical pulps. BioResources 9:2115-2127

Le Baillif M, Oksman K (2009) The effect of processing on fiber dispersion, fiber length and thermal degradation of bleached sulfite cellulose fiber polypropylene composites. J Thermoplast Compos 22:115-122

Luner PE, Oh E (2001) Characterization of the surface free energy of cellulose ether films. Colloid Surf A 181:31-48

Morales JO, McConville JT (2011) Manufacture and characterization of mucoadhesive buccal films. Eur J Pharm Biopharm 77:187-199 
Nechyporchuk O, Pignon F, Belgacem MN (2015) Morphological properties of nanofibrillated cellulose produced using wet grinding as an ultimate fibrillation process. J Mater Sci 50:531-541

Osong SH, Norgren S, Engstrand P (2016) Processing of woodbased microfibrillated cellulose and nanofibrillated cellulose, and applications relating to papermaking: a review. Cellulose 23:93-123

Rahmat D, Sakloetsakun D, Shahnaz G, Perera G, Kaindl R, Bernkop-Schnuerch A (2011) Design and synthesis of a novel cationic thiolated polymer. Int J Pharm 411:10-17

Rol F, Karakashov B, Nechyporchuk O, Terrien M, Meyer V et al (2017) Pilot-scale twin screw extrusion and chemical pretreatment as an energy-efficient method for the production of nanofibrillated cellulose at high solid content. ACS Sustain Chem Eng 5:6524-6531

Sehaqui H, Zhou Q, Berglund LA (2011) Nanostructured biocomposites of high toughness-a wood cellulose nanofiber. Soft Matter 7:7342-7350

Simão CD, Reparaz JS, Wagner MR, Graczykowski B, Kreuzer $M$ et al (2015) Optical and mechanical properties of nanofibrillated cellulose: toward a robust platform for nextgeneration green technologies. Carbohydr Polym 126:40-46

Siró I, Plackett D (2010) Microfibrillated cellulose and new nanocomposite materials: a review. Cellulose 17:459-494
Sirviö JA, Heiskanen JP (2017) Synthesis of alkaline-soluble cellulose methyl carbamate using a reactive deep eutectic solvent. ChemSusChem 10:455-460

Spence KL, Venditti RA, Rojas OJ, Habibi Y, Pawlak JJ (2011) A comparative study of energy consumption and physical properties of microfibrillated cellulose produced by different processing methods. Cellulose 18:1097-1111

Suzuki K, Okumura H, Kitagawa K, Sato S, Nakagaito AN, Yano H (2013) Development of continuous process enabling nanofibrillation of pulp and melt compounding. Cellulose 20:201-210

Taheri H, Samyn P (2016) Effect of homogenization (microfluidization) process parameters in mechanical production of micro- and nanofibrillated cellulose on its rheological and morphological properties. Cellulose 23:1221-1238

Toba K, Yamamoto H, Yoshida M (2012) Mechanical interaction between cellulose microfibrils and matrix substances in wood cell walls induced by repeated wet-and-dry treatment. Cellulose 19:1405-1412

Wu CJ, Zhang CJ, Yu DM, Li RG (2018) Dissolving pulp from bamboo-willow. Cellulose 25:777-785

Publisher's Note Springer Nature remains neutral with regard to jurisdictional claims in published maps and institutional affiliations. 